\title{
AGRICULTURA Y DESARROLLO DEL ESTADO EN LAS TIERRAS BAJAS MAYAS*
}

\author{
B. L. Turner II \\ Universidad de Oklahoma
}

Numerosas tesis sobre el Estado o el desarrollo sociopolítico han sido formuladas dirigiéndose, tanto en lo general como en lo específico, al caso de la civilización maya de las tierras bajas. Entre éstas, la tesis hidráulica ha tenido un impacto considerable al intentar explicar los diferentes niveles del crecimiento del Estado logrados por los mayas de las tierras bajas y las culturas mexicanas de las tierras altas durante tiempos prehispánicos. En resumen, los que proponen la tesis hidráulica o sus elaboraciones asumen que las dicotomías en el desarrollo del Estado entre las civilizaciones de las tierras altas y las de las tierras bajas eran, en parte, el resultado de diferentes tecnologías agrícolas y/o factores del medio geográfico en las dos regiones. Las evidencias recientes de la agricultura maya prehispánica indican que las diferencias propuestas en tecnología agrícolas pudieron no haber exsitido y que las tierras bajas ofrecían un medio ideal para cultivos. En este trabajo: i) se discute brevemente la evidencia reciente de la agricultura antigua en las tierras bajas mayas, ii) se examinan algunos de los problemas creados por la evidencia de dos tesis hidráulicas relacionadas que han sido aplicadas a los mayas y iii) se ofrecen los rudimentos de un argumento alternativo para explicar el nivel relativo alcanzado en las tierras bajas, en cuanto al desarrollo del Estado.

Esta discusión se centra en varios argumentos que tratan de expli. car la diferenciación sociopolítica entre las civilizaciones maya y del Centro de México, específicamente Teotihuacán, y no tiene qué ver

* Traducción del trabajo presentado en el Simposio de Agrosistemas Mexicanos: Pasado y Presente. XLIII Congreso Internacional de Amer'canictas (traducción de Rebeca González revisada por la Comisión Editorial del Centro de Estudios Mayas). 
con la controversia alrededor de la definición de Estado o el nivel preciso de desarrollo socio-político que pudo haber sido alcanzado por los antiguos mayas. La noción del crecimiento del Estado es utilizado libremente para referirse al desarrollo de controles sociopolíticos centralizados en una sociedad estratificada. Para facilitar la discusión se acepta que la civilización clásica maya no alcanzó el mismo nivel de crecimiento estatal que el de las culturas del Centro de México, aunque en el futuro los estudios podrían no verificar este punto de vista. Se enfatiza aquí que diferencias aparentes en el desarrollo del Estado entre los habitantes de las tierras altas y los de las tierras bajas, pue. den no ser explicados adecuadamente por las tesis aquí explicadas. Los argumentos recientes desarrollados por William T. Sanders y por Bárbara J. Price han sido seleccionados para ser examinados porque éstos representan una corriente de pensamiento de dos de los más influyentes y elocuentes estudiosos en el campo de la prehistoria meso. americana, que han dedicado gran atención a la tesis hidráulica. Los comentarios dirigidos a estos trabajos son ofrecidos dentro de un espíritu de diálogo intelectual.

\section{Agricultura maya prehispánica}

Las crecientes evidencias indican que los antiguos mayas de las tierras bajas utilizaron varias formas avanzadas de agricultura para cultivar intensivamente numerosos microambientes. Vestigios de por lo menos tres antiguos métodos de cultivo se presentan tan sólo en el sur de Campeche y Quintana Roo, México, incluyendo terrazas, redes de irrigación y campos levantados. El sistema de terrazas se continuó usando por los mayas a través de las serranías interiores en forma de laderas lineales, terrazas de cultivo sin riego y terrazas de fondo acanalado (Turner, 1974; 1978: Figs. 7.2 y $7.3 ;^{*} 1979$ ). Esta técnica de cultivo fue utilizada sobre terrenos de poca inclinación, especialmente en la zona de Río Bec, para impedir la erosión, para establecer el grosor del suelo y para incrementar la humedad de éste por la captura de desagües. Una probable red de irrigación ha sido identificada en Edzná, Campeche, que fue utilizada para la agricultura y usos domésticos (Matheny, 1976). La agricultura de campos levantados, una forma hidráulica de cultivo, fue utilizada en zonas ribereñas y en depresiones temporalmente inundadas en el sureste de Campeche, sur de Quintana Roo y norte de Belice (Siemens y Puleston, 1972; Turner,

* Nota del editor. 
1974; Harrison, 1977). Este tipo de agricultura imponía la construcción de plataformas y canales de tierra y roca intemperizada que daban protección en caso de inundación durante la temporada de lluvias y, posiblemente, irrigación para el cultivo en la estación de secas, similares al sistema de chinampas del Valle de México (Denevan y Turner, 1974; Gómez Pompa, 1974; 1978). Además, otros vestigios probablemente asociados con agricultura, sugieren la utilización intensiva de la tierra. Quizá los más importantes de estos elementos son las numerosas paredes de piedra que cubren grandes sectores de Campeche y Quintana Roo, delimitando la tierra en pequeños campos rectangulares (Turner, 1974; Sabloff y Rathje, 1975; Eaton, 1975; Thomas, 1976; Vleck et al., 1978). Encontradas con frecuencia en laderas terraceadas, estas paredes no tienen función conocida pero sugieren una utilización intensiva de la tierra en el pasado (Turner y Harrison, 1978).

Evidencias de agricultura maya intensiva y manejo del suelo, algunas de las cuales eran hidráulicas, también se encuentran en los alrededores inmediatos de México. Antiguos campos levantados se extienden hacia el sur, a lo largo los ríos Hondo y Nuevo Belice en la vecindad de San Antonio y aún más lejos (Siemens, 1977; Puleston, 1977). Algunas de las depresiones en el Petén Central podrían dar señales de manejo del agua en el pasado (Back y Hanshaw, 1967). Las terrazas se presentan al pie de las montañas mayas (Rice, 1974; Turner, 1979; Healey et al., s/f) y en el Petén, podrían ser más frecuentes de lo que habíamos pensado (Turner, 1974a; Turner y Harrison, 1978; Rice y Rice, 1978). "Planitos" o cortes en las laderas hechas por los mayas ocurren en la región de Copán (Turner y Johnson, s/f). Antiguas presas mayas fueron reportadas recientemente en esa región (Turner y Johnson, 1979) y posiblemente en el Petén (Rice y Rice, s/f). Así mismo se sabe que, en algunos sitios mayas tenían depósitos y sistemas de distribución de agua para usos domésticos, como los de Tikal y Copán.

Estos vestigios han sido discutidos en detalle en otro lado y no necesitan ser reiterados ampliamente (Harrison y Turner, 1978). La importancia de estas cvidencias es que indican que: i) los antiguos mayas de las tierras bajas conocían y utilizaban virtualmente todas las formas de cultivo y técnicas de control de agua conocidos por los amerindios; ii) que muchas de estas técnicas de agricultura intensiva representan una alta entrada y salida de energía y iii) que el uso de estas técnicas, en extensas áreas, probablemente sobrepasan a cualquier otra región de Mesoamérica. Los campos levantados, por ejemplo, probablemente ocurrieron a través de continuas zonas ribereñas en Quin- 
tana Roo y Belice que excedieron los $450 \mathrm{kms}^{2}$ (Turner y Harrison, 1978: $338-41$ ). Esta evidencia implica serias interrogantes a aquellos planteamientos que argumentan una causa hidráulica relacionada con el desarrollo del Estado en las tierras altas de México en el altiplano mexicano pero no en el caso de las tierras bajas del área maya.

\section{Tesis hidráulica sobre el desarrollo del Estado}

La tesis hidráulica sobre el desarrollo del Estado fue elaborada primero por Wittfogel (1957). En síntesis, esta tesis sostiene quo los altos niveles de desarrollo del Estado resultan del control centralizado de las actividades comprendidas en la agricultura hidráulica; especialmente la agricultura de irrigación a gran escala. El nivel de desarrollo está relacionado al significado de los cultivos hidráulicos en la producción total de alimentos en oposición a cultivos temporales $u$ otras formas de cultivar. Ya que las regiones áridas tienden a necesitar obras hiçráulicas para tales fines, por lo tanto aquellas culturas que ocuparon esas regiones tuvieron una tendencia a desarrollarse en sociedades estatales complejas (o sociedades hidráulicas compactas).

Aunque los principios básicos de la tesis hidráulica han llamado bastante la atención entre numerosos mesoamericanistas, probablemen. te Sanders y Price han dedicado mucha más atención al tema y han proporcionado mayores contribuciones para la aplicabilidad de la tesis, o de sus modificaciones, que cualquier otro. En su importante trabajo Mesoamerica, The Evolution of Civilization, Sanders y Price establecieron los fundamentos para sus futuros argumentos, concluyendo que el desarrollo del Estado (y el urbanismo) en el centro de México fue producto de la interacción entre el crecimiento de población, com. petencia y cooperación en un marco ambiental único (1967: 191-93). Uno de los resultados de esta interacción fue la agricultura hidráulica, factor que ayudó a promover las interacciones y crecimiento del Estado. En contraste, se pensó que los mayas de las tierras bajas estaban limitados a la agricultura de roza, un factor no conducente en las necesarias interacciones para el desarrollo del Estado. Los logros materiales de los mayas y su limitado desarrollo sociopolítico fueron explicados en términos de contactos e influencias del centro de México.

La tesis de Sanders y Price fue muy estimulante, pero fue propuesta en una época en que el conocimiento de la agricultura maya prehispánica estaba limitado y basado en gran parte en suposiciones (Turner, 1978a). Los que propusieron esta tesis subsecuentemente han modificado y elaborado el argumento de acuerdo a las críticas y la nueva 
evidencia. Los últimos esfuerzos explicatorios de Sanders y Price pueden ser vistos como progresos temporales e intelectuales de su trabajo original. La diferenciación agrícola que se ha propuesto entre las civilizaciones del altiplano y las de las tierras bajas queda como un elemento importante de esos argumentos.

La última ocntribución de Sanders al tema (1977), enfatiza la heterogeneidad de los hábitats de las tierras bajas en términos de las características del suelo y el potencial de cada tipo de suelo para la agricultura maya. El concluye que la región tenía un alto potencial para cultivo de roza, pero un potencial variable para la agricultura intensiva. Se reconoce la evidencia de las tierras bajas, pero se ve representando sistemas de agricultura intensiva, restringidos espacialmente, que no podían expanderse a causa de factores ambientales. Sanders concluye que tales restricciones promovieron la centralización sociopolítica en las tierras bajas hasta cierto nivel. El desarrollo de un Estado verdadero no se logró ya sea porque los sistemas agrícolas eran inadecuados, o porque los hábitats locales no pudieron sostener el proceso de intensificación agrícola.

Estas conclusiones son compatibles con los anteriores trabajos de Sanders, pero el argumento ha sido modificado. Previamente él había enfatizado la heterogeneidad ambiental en el altiplano y la homogeneidad de las tierras bajas (en comparación a las tierras altas), como una explicación parcial de las discrepancias en cuanto al desarrollo del Estado en las dos regiones (Sanders, 1972). Este argumento reciente reconoce correctamente que las tierras bajas tienen diversidad de hábitats, pero concluye que la mayoría de éstos no fueron adecuados para la agricultura intensiva. Si la agricultura intensiva se refiere o no a cultivos hidráulicos, no está claro.

Numerosos problemas se enfrentan en cualquier intento por evaluar las cualidades del suelo en las tierras bajas, y no son menos los que conciernen a la cantidad y calidad de los datos. Sanders está consciente de los problemas de los datos, pero son necesarios varios comentarios en lo concerniente a la evolución de los suelos del Petén, sobre los que basó sus argumentos. El mapa de suelos elaborado por Simmons y sus colegas (1959) está basado en el análisis de la flora obtenido de fotografías aéreas. Gran parte de la distribución de suelos propuesta no fue verificada en tierra, y el papel que jugaron las modificaciones humanas sobre la vegetación y cómo este factor fue considerado por el equipo de estudio, no fue adecuadamente explicado. Los estudios de los suelos en la región, sin embargo, han verificado los patrones generales de erosión y calidad de drenaje demarcados en el trabajo original, y se ha demostrado que las tierras bajas mayas mantienen una mezcla 
compleja de suelos, muchos de los cuales son fértiles para el cultivo (Olson, 1969; 1975; Turner, 1978b; Turner y Johnson, 1978). Aparte de los problemas con los datos, deberían de ser planteadas serias interrogantes en lo que concierne a cualquier intento de establecer el potencial del medio para la agricultura, como quiera que se le defina. Tales potenciales pueden ser evaluados sólo si se acepta que el medio es el principal agente que influye en el desarrollo agrícola. Numerosos estudios han indicado que esta circunstancia no es básica, sino que varios factores como la demanda de producción y tecnología, parte de la cual mantiene una relación de interacción con el hábitat físico, constituyen las principales influencias en el desarrollo agrícola (Brook field, 1972; Turner et al., 1977). Dada esta evidencia, probablemente deberían enfocarse avalúos agrícolas de los hábitats sobre las posibi. lidades del medio o la adaptabilidad del hábitat a distintos niveles de producción agrícola cuando son aplicadas diversas tecnologías. En este sentido, el medio restringe la agricultura en términos de eficiencia, pero no en su crecimiento (Brookfield, 1972). Para dictaminar los usos agrícolas del suelo es necesario entender los numerosos factores críticos, particularmente la tecnología que fue o podría haber sido empleada. Sanders aparentemente concluye que los mayas estuvieron limitados por condiciones físicas locales, principalmente los suelos, utilizando técnicas agrícolas intensivas, no en todos, sino en algunos lugares. Los sistemas del tipo de roza predominaron en las tierras bajas. Este punto de vista solamente puede ser sostenido si se asume que los hábitats, en última instancia, determinan el desarrollo agrícola $\mathrm{y} / \mathrm{o}$ dando interpretaciones mínimas a los recientes datos agrícolas.

Como fue sugerido anteriormente, los mayas prehispánicos tenían la habilidad y tecnología para dedicarse al cultivo intensivo e hidráulico en toda la extensión de las tierras bajas. Parte de la evidencia que sostiene este punto de vista está en oposición directa con las zonas potenciales de suelos propuesta por Sanders. El grupo de suelos IIb,* caracterizado por poca profundidad, bien drenado y cuya fertilidad tiende de moderada a alta, son en su mayoría mollisols, de calidad mediana para cultivos, pero con problemas de erosión (ver Turner, 1978b). Aparentemente, Sanders asume que la intensificación agrícola en este tipo de suelos daría lugar a una baja en rendimiento y, por lo tanto, habría malogrado el crecimiento agrícola. Es interesante seña. lar que terrazas a gran escala diseñadas para contrarrestar los problemas de erosión, fueron construidas por los mayas prehispánicos en suelos del grupo IIb, inmediatamente al norte (en el sur de Campe.

* Cf. Sanders, 1977. (N. del E.) 
che y Quintana Roo) y al este (en Belice) del área considerada por Sanders. Otro conflicto se presenta en los suelos del grupo IIc, caracterizados como profundos, pobremente drenados, de fertilidad baja a alta y poco erosionables. Estos suelos que son predominantemente vertisols y mollisols en el norte del Petén, fueron clasificados virtualmente como no potenciales para la agricultura maya, presumiblemente por los problemas de drenaje. Otra vez, la continuación de la zona de suelos IIc en el sector noreste del Petén, hacia Quintana Roo y Belice, nos conduce a extensas redes de antiguos campos levantados; vestigios de una agricultura hidráulica intensiva.

La poca evidencia de terrazas y campos levantados en los grupos de suelo IIb y IIc, respectivamente, en el Petén, no puede ser explicada en términos de limitaciones ambientales locales o tecnología agrícola inadecuada. Es claro que las dos zonas de suelos fueron utilizadas para cultivos intensivos en las cercancías del Petén. Las razones por las cuales terrazas y campos levantados fueron utilizados en algunas áreas de este tipo de suelo, y no en otras, es una pregunta intrigante que probablemente se relacione con las decisiones socioeconómicas de los mayas, aunque las características hidrológicas de las tierras inundables en el área maya, podrían haber constituido un factor que influyó en el emplazamiento de los campos levantados (Siemens, 1978).

La utilización agrícola de la mayor parte de los suelos de las tierras bajas no fue inhibida por los niveles de fertilidad propia. Así como el 83\% del Petén está cubierto por suelos de fertilidad que tiende de moderada a alta, el sur de Campeche y Quintana Roo en compara. ción, no está mal, Simmons et al., 1959; Turner, 1978b). Los agricultores mayas aparentemente tenían la habilidad de mantener la fertilidad de los suelos con el uso de varias técnicas. Los agricultores prehispánicos que en Mesoamérica utilizaron formas similares de agricultura a la de los mayas, se sabe que emplearon varias fuentes de fertilizantes y técnicas para mantener la fertilidad, tales como cubrir las plantas con estiércol y paja para protegerlas, abonar (a menudo con heces humanas) y rehabilitación de los suelos. Fuentes y procedimientos similares fueron sin duda accesibles a los mayas, incluyendo abono de origen animal (Turner y Harrison, 1978). Es muy difícil asumir el punto de vista de que pueblos que participaban en prácticas agrícolas semejantes no compartieran habilidades similares para manipular los suelos, particularmente dado el hecho de que agricultores menos avanzados técnicamente que los mayas usaban numerosos procedi. mientos para mantener los suelos (Brookfield, 1972; Wilke, 1977a y b).

La evidencia no sostiene el punto de vista de que los mayas estaban 
limitados al cultivo de roza; que no practicaban cultivos hidráulicos, - que los factores ambientales impedían el desarrollo agrícola inten. sivo en las tierras bajas. Las tecnologías en las tierras altas y en las tierras bajas eran básicamente las mismas. La diferencia más grande entre los agrosistemas de las dos regiones incluye la necesidad de riego artificial para el cultivo.

La irrigación en las tierras secas fue necesaria en muchas secciones de los valles del Altiplano para garantizar un margen de seguridad a la agricultura intensiva. En contraposición, con un aceptable margen de seguridad, gran parte de las tierras bajas centrales de los mayas, probablemente pudieran haber tenido cultivos intensivos sin irrigación te tierras secas, aunque la técnica podría haber sido utilizada. La apa. rente dicotomía entre la necesidad y el uso de irrigación de tierras secas que por lo tanto se ha supuesto en las dos regiones, es un punto de gran importancia adelantado por Price.

En su reciente modelo de interacción-agrupamiento, Price (1977) sostiene que el crecimiento sociopolítico ocurre en asociación con los incrementos en la productividad, población y contenido de energía de sistemas, y que la irrigación de las tierras secas representa un cambio en el modo de producción que promueve estos incrementos. Los alrededores de Teotihuacán son vistos como situados idealmente para el desarrollo de un sistema extensivo de agricultura hidráulica, en el cual los pantanos alimentados por manantiales fueron convertidos en chinampas y manipulación por canales de riego. Esta forma de agricultura, aceptada en su más amplio contexto hidráulico ayudó a estimular el crecimiento del Estado porque constituía un cambio en el modo de producción.

En contraste con los habitantes del Altiplano, los mayas se caracterizaban como esencialmente agricultores de roza que intensificaron la producción primeramente reduciendo el periodo de barbecho. Jardines-huertos, campos levantados y agricultura en pantanos permitían intensificar la producción, pero estos procedimientos no condujeron a cambios en el modo de producción. El crecimiento sociopolítico en las tierras bajas está explicado en términos de contacto y comercio con Estados cercanos, siguiendo las ideas de Wittfogel de sociedades hidráulicas marginales.

Este argumento padece de varias inconsistencias. En efecto, los mayas son descritos como básicamente agricultores de roza, aun cuando la riqueza de la información sugiere otras explicaciones. Aunque la información sobre terrazas e irrigación es ignorada ( $o$, por lo menos, no está tomada en cuenta), la intensificación de la producción por uso de jardines-huertos y campos levantados, es reconocida. Curiosamente, 
a los campos levantados de los mayas no se les otorga el mismo status que a los campos "propuestos" en Teotihuacán. Ambos campos, los de la altiplanicie y los de las tierras bajas, fueron presumiblemente construidos para drenar tierras inundadas y proveer de un constante régimen de humedad para la agricultura intensiva, pero el primero implicaba un cambio en el modo de producción y el último no.

La tecnología de campos levantados es básicamente la misma en todo el mundo, particularmente cuando es utilizada en hábitats similares (Denevan y Turner, 1974). Diferencias funcionales o productivas en la tecnología no pueden ser inferidas de las taxonomías locales, tales como chinampas, campos levantados o polders. En verdad, las funciones inferidas de las situaciones de técnicas-hábitats descritas para Teotihuacán y la región maya parecen ser bastante similares. De cualquier modo, Price relega el significado de los campos levantados mayas para hacer notar correctamente que esta forma de cultivo es practicada en Nueva Guinea por grupos igualitarios (Brookfield y Brown, 1963; Waddell, 1972). Ella no aplica esta analogía a los campos levantados de Teotihuacán. Este razonamiento está aparentemente legitimizado en los terrenos que, al igual que en los campos que se localizan cerca de Teotihuacán, ocurren "como parte de un contexto hidráulicos más general en el cual todas las partes se refuerzan" (Price, 1977: p. 215). El propósito de esta declaración no es claro, pero un argumento que puede ser fácilmente propuesto es que los campos mayas se daban en contextos hidráulicos similares a los propuestos para los campos de la altiplanicie. Los mayas utilizaban va. rios métodos de control del agua, incluyendo canalización a depresiones donde estaban los campos y, tal vez, regulación interior de los ni. veles de agua de las depresiones. Algunas depresiones de las tierras bajas, especialmente aquéllas del sur de Quintana Roo, parecen tener intrincadas redes de grandes y pequeños canales dispersos entre los campos levantados, sugiriendo una función de control de agua. Los estudios recientes de la Universidad de Oklahoma, ${ }^{*}$ indican que el mantenimiento constante de los niveles de agua en los canales de los campos levantados tuvo una función mayor que en los campos de las tierras bajas (Turner, Harrison et al., s/f). Esta función es idéntica a la de los sistemas de chinampas del Altiplano.

El "contexto hidráulico" de Price parece referirse a la supuesta presencia de irrigación de tierras secas asociadas con los campos de Teotihuacán. En el medio semiárido de la altiplanicie', los canales asociados a los campos se piensa que canalizaban agua a las superficies le-

* N. S. F. Pulltrouser Swamp Project. 
vantadas. Se puede argumentar que los grandes y pequeños canales que entrelazan los campos levantados mayas, tenían una función similar a la asignada por Price a los campos de Teotihuacán, especialmente para cuitivos en la temporada de secas o tiempos de sequía. La evidencia de "irrigación por canales" de los campos levantados antes de o durante el periodo Clásico (antes de 900 d. C.) en la vecindad de Teotihuacán o en el área maya es meramente especulativa. Debe de reconocerse que los pequeños complejos de campos levantados que se extienden cerca de Teotihuacán no han sido fechados para épocas prehispánicas, pero existen fuertes razones para pensar que se trata de campos antiguos. Sin embargo, la evidencia indica que la irrigación fue practicada en el área de Teotihuacán en una época no determina. da antes del Post-clásico (1350-1519 d. C.) (Sanders, Parsons, Santtley, 1979) y por lo menos un complejo de campos levantados en el área maya se ha sugerido que tiene una fecha del Formativo (Puleston, 1977).

La prevaleciente necesidad de tener mayor irrigación en las tierras secas de la altiplanicie árida que en las tierras bajas centrales mayas, queda como la principal diferencia agrícola entre ambas regiones. La evidencia histórica, e inferencias de ella, sugiere que tal tecnología era probablemente más importante a la agricultura en los contornos del Valle de México, fuera de los lagos interiores, que en la región maya. Aceptando esta dicotomía inferida, los rasgos singulares asociados con irrigación que conducen a control centralizado (cambio en modo de producción) y crecimiento sociopolítico, requieren identificación. En este sentido, Price exhibe otra inconsistencia en la interpretación de la información. Con base en la evidencia de Nueva Guinea (e. g., Brookfield y Brown, 1963), Price rechaza el papel de la agricultura de campos levantados como el que impulsa a un cambio en el modo de producción. La evidencia de que la irrigación tal vez no permite tal cam. bio, especialmente en cuanto al desarrollo del Estado, se descarta con poca consideración. Este tema merece un análisis mayor.

Estrictamente interpretada, la solidez de la tesis hidráulica descansa en la demostración de la relación causal entre la irrigación y el desarrollo sociopolítico. La causa podría ser inferida demostrando que para la irrigación a gran escala se necesitan controles a nivel de Estado. Adams (1960), Hole (1966), y Lanning (1967), entre otros, han concluido que los controles estatales centralizados anteceden a operaciones de irrigación a gran escala en Mesopotamia, Mesoamérica y en la región de los Andes. El estudio comparativo de Kappel (1974) da apcyo a las implicaciones de esta conclusión, sugiriendo que aparecen cambios en los sistemas de irrigación después de que ocurren cambios 
del crecimiento sociopolítico. Aparte de la cuestión temporal, Butzer (1976) y Leach (1959) demuestran convincentemente que los extensos y complejos sistemas de irrigación del Egipto faraónico y Geilán antiguo, respectivamente, fueron construidos y mantenidos por políticas locales, no estatales. También Mitchell (1977) ha establecido que "procedimientos habituales" (controles locales) gobiernan el control de irrigación en Quinua, Perú, y que fuerzas políticas (centralizałas) sólo legitimizan los reconocidos controles locales.

Las causas no necesariamente infieren una secuencia temporal de eventos, pero generalmente requieren que pueda ser postulada una relación funcional o interaccional. Como Mitchell hace notar (1973: 533) la tesis hidráulica implica que debería esperarse que irrigación y control" político se desarrollaron juntos, "interactuando en una forma sinérgica" (mecanismos de retroalimentación). Esta declaración sugiere que debe ser esperada una relación directa entre el tamaño o el significado de la irrigación y grado de desarrollo del Estado. El estudio de Millon (1962) de sistemas de irrigación a pequeña escala indica que en tales sistemas los conflictos y la competencia por el agua dan como resultado controles sociopolíticos. La centralización de estos controles, sin embargo, no tiene una relación clara con el tamaño del sistema de irrigación o el significado en términos del tamaño de la población que sostenía. Reconociendo estos problemas, Mitchell (1973: 534) ha formulado la tesis hidráulica: "si hay dirección centralizada de las actividades de irrigación en un medio árido o semiárido, entonces habrá un incremento en la correspondencia, en la fuerza política centralizada y otras esferas de la vida social". No es explicado el papel que juegan los medios áridos o semiáridos.

En respuesta a estos temas Price sostiene que la relación entre agricultura hidráulica y el desarrollo del Estado no es de simple causalidad lineal. Como quiera que sea, su alternativa no es clara. Inicialmente ella sostiene que un enfoque superior es el de un sistema en el cual la irrigación es solamente uno de los varios componentes que interactúan y se refuerzan recíprocamente (Price, 1977: 215). Más adelante ella argumenta que si fuera establecida una relación funcional entre obras hidráulicas y poder estatal, entonces las causas podrían ser inferidas lógicamente (Price, 1977: 216). El último argumento no ha sido demostrado y la adopción del primero reduce la fuerza explicativa del factor hidráulico. Aparentemente, Price ve la irrigación como una condición necesaria pero no suficiente para estimular el crecimiento estatal. Las características de la irrigación son la competencia y cooperación que resulta por la necesidad del agua. La evidencia de que estas características deriven sólo de la agricultura hidráulica y no 
de otras técnicas de cultivo no ha sido documentada adecuadamente, no importa qué tan intuitivamente fuerte aparezca este avalúo. Estas consideraciones indudablemente influyeron en la reformulación de la tesis hidráulica de Mitchell. Sin embargo, la explicación del desarrollo sociopolítico se hace rechazando aquellos "...factores que predisponen a ciertas sociedades a desarrollar directrices centralizadas de las obras hidráulicas" (Mitchell, 1973: 534). Hasta que sea demostrado que sólo las técnicas de irrigación estimulan la cooperación y competencia, las últimas palabras de esa oración pueden ser eliminadas.

\section{Aclaraciones, modificaciones e investigaciones mesoamericanas}

La aplicación de la tesis hidráulica y sus derivados para explicar el florecimiento de los Estados en Mesoamérica se ha topado con serios problemas de datos y conceptos. Estos problemas arrojan dudas en los intentos de explicar las discrepancias propuestas en el desarrollo del Estado en la altiplanicie mexicana central y las tierras bajas mayas en términos de información hidráulica y agrícola. Por lo menos tres enfoques, implícitos y explícitos, han sido identificados como intentos por evadir estos problemas: i) gran parte de las evidencias pertenecientes a la agricultura maya prehispánica son ignoradas o relegadas, ii) técnicas agrícolas similares son asignadas a diferentes implicaciones sociopoliticas, y iiii) se hace referencia a factores ambientales que se piensa que limitaron el desarrollo agrícola.

Se sugiere que muchos de estos intentos, dada la evidencia, son explicaciones inadecuadas. El significado inferido de la irrigación de las tierras secas en los valles centrales mexicanos parece ser la clave de la distinción agrícola entre el Altiplano y las tierras bajas, aunque no es sino hasta ahora que la presencia y significado de la irrigación entre los mayas se ha manifestado. Otras cualidades agrícolas entre las dos regiones y sus habitantes, tales como capacidad tecnologica, producción y estabilidad, serían probablemente comparables. De esta manera, la búsqueda de una explicación "hidráulica" de las discrepancias propuestas del desarrollo sociopolítico en Mesoamérica, debería ser enfocada hacia investigaciones dirigidas: i) a la demostración de que las distinciones y el significado de la irrigación de las tierras secas entre la altiplanicie central mexicana y la de las tierras bajas realmente existía y, ii) fuera de la subordinación de argumentos que degradan las habilidades agrícolas de los mayas o que enfatizan la marginación del medio de las tierras bajas para la agricultura. Los datos sugieren que los argumentos orientados hacia lo hidráulico se revierten 
a la controversia original alrededor de supuestas consecuencias sociopolíticas o de irrigación de la tesis de Wittfogel.

En el futuro, el enfoque hidráulico deberá resolver varias cuestiones concernientes a la agricultura maya. ¿En qué extensión practicaron los mayas la irrigación de las tierras secas y qué tan significativa fue esta forma de agricultura? La evidencia de Edzná y los sistemas de irrigación/drenaje para usos domésticos en Tikal y Copán, sugieren que la irrigación de las tierras secas podría haber sido más importante de lo que se pensó previamente. ¿ Regulaban los mayas el flujo del agua en depresiones (bajos) con los campos levantados de tal manera que inundaciones y control de irrigación eran cruciales para la producción agrícola? La competencia y cooperación de estos controles de agua podrían haber tenido los mismos efectos sociopolíticos que han sido sugeridos para la irrigación de las tierras secas. ¿Controlaban los mayas las inundaciones de las depresiones, sin necesidad de recurrir a los campos levantados, para uso doméstico o para crear fertilizantes ricos en nutrientes de limo? La técnica principal en la fertilización de los suelos en cultivos de relleno son las inundaciones controladas, y este procedimiento parece fácilmente adaptable en las depresiones de las tierras bajas mayas.

Tales indagaciones pueden conducirnos a la conclusión de que la tecnología agrícola entraría mejor en la categoría de obras hidráulicas, llamadas de "función directa de producción", en vez de en la categoría de "función indirecta de producción" asignadas por Wittfogel (1972: 67). En efecto, la evidencia sugiere tentativamente que la agricultura maya cabe mejor dentro de la clasificación de ríos inundables y lagos (depresiones), equiparando esta civilización con las de Egipto e India en términos de las implicaciones sociopolíticas del esquema de Wittfogel. Claro está que la regulación de niveles de agua en las depresiones de las tierras bajas podría haber sido un logro hidráulico más sofisticado que requería una coordinación más centralizada que la irrigación por inundación en el Valle del Nilo. Indiferente a la función precisa de las obras hidráulicas de los mayas, la evidencia indica que la tesis hidráulica necesita una reevaluación considerable en términos de la civilización de las tierras bajas, como lo ha señalado Matheny (1978).

La reclasificación de los mayas dentro de una categoría hidráulica superior no resolvería los problemas fundamentales asociados con la tesis hidráulica, especialmente cuando la evidencia de que el crecimiento del Estado despótico en el antiguo Egipto no involucraba una relación directa con el control de la irrigación de las vegas aluviales (Butzer, 1976). Esta evidencia sugiere que los argumentos alternos 
podrían ser explorados como explicaciones de la información de los mayas y de los mesoamericanos. Investigaciones recientes sobre el crecimiento agrícola entre agricultores a nivel de subsistencia, dan un marco de trabajo para el establecimiento de varias alternativas. Estos estudios sugieren que la tecnología agrícola, incluyendo irrigación, es una manifestación de la presión o tensión de los niveles de producción creados por la interacción de la demanda para la producción agrícolas con variables ambientales y otras (Boserup, 1965; Brookfield, 1972; Turn r et al., 1977). A medida que la presión de la producción se incremerta por unidad de área, los agricultores deberán intensificar el rendimiento $y$, por lo tanto, adoptar o desarrollar una tecnología que permita que la producción se incremente. La tecnología específica utilizada resulta principalmente de los factores ambientales y los diversos cultivos básicos. Los factores ambientales son especialmente importantes en esta relación, de tal manera que un nivel constante de demanda de producción creará varios niveles de tensión sobre una extensión de medios físicos, variando la calidad de las condiciones de cultivo, de pobre a alta.

Aunque peligroso, es tentador extrapolar los modelos de desarrollo agrícola a las inferencias sociopolíticas. Se ha supuesto que los altos niveles de tensión crean niveles altos de desarrollo sociopolítico (e. g., Carneiro, 1970). Las cualidades y especificidades de tal tensión han sido demasiado simplificadas y no han sido elaboradas completamente. Por lo menos son sugeridas dos modificaciones del argumento de tensión para los modelos de crecimiento agrícola. Estas manipulaciones se relacionan con las consecuencias de interacción entre población y el medio, y las causas del incremento de la presión de la producción. En el primero, las modificaciones en cada hábitat físico está evaluado en términos de su posibilidad o facilidad para cultivos, clasificados de marginal a óptimo. Medios bastante marginales necesitan invertir más energía por unidad de rendimiento de lo que necesitan para su desarrollo los medios óptimos, resultando en altos niveles de presión productiva con pequeñas poblaciones de baja densidad. Igualmente, las condiciones óptimas para cultivos que están limitadas en su extensión podrían sucumbir rápidamente a la tensión porque es más fácil inten. sificar el incremento en ese tipo de terreno que cultivar terrenos ad. yacentes pero más marginales, un argumento similar al concepto propuesto por Carneiro de circunscripción ambiental. La segunda modificación concierne a los elementos "circunscriptores" que crean tensión y a las consecuencias sociopolíticas. Los grupos que han llegado a los límites del área bajo su dominio y están imposibilitados de expander su territorio agrícola pueden ser vistos como incurriendo en una ten- 
sión "legítima"; de ello pueden resultar controles sociopolíticos por la competencia para la producción (ya sea producción nativa o comercio). Sin embargo, pueden ocurrir situaciones en las cuales la tensión es inducida "artificialmente", quizá como ocurre en Nueva Guinea, por una decisión de intensificar la producción más que por cultivar una gran área. En tales circunstancias, el desarrollo sociopolítico quizá no podría llevarse a cabo, o ser impedido porque los intentos por controlar la producción pueden ser evitados por el cambio hacia tierras vírgenes.

Estos argumentos tienen implicaciones para los mayas y otras civilizaciones mesoamericanas. Los mayas tal vez nunca llegaron a los mismos niveles de tensión como sucedió en algunos de los valle del Altiplano a causa de la gran cantidad de suelos de calidad moderada a óptima disponibles para su cultivo en las tierras bajas (Turner y Harrison, 1978: 361-71). Los incrementos en la tensión pudieron haber sido aliviados por el cultivo de nuevos terrenos, a menos que se toparan con niveles substanciales de población. En contraste', los valles en el Altiplano a menudo contenían cantidades limitadas de tierras óptimas a moderadas para cultivar, de manera que los niveles altos de tensión podrían haber sido encontrados en poblaciones relativamente pequeñas. Las dicotomías en tensión pueden haber dado como resulta. do dicotomías en el desarrollo del Estado. El crecimiento sociopolítico en las tierras bajas mayas no estuvo limitado por factores ambientales o modos de producción, pero pudo haber sido influido por la abundancia de hábitats adecuados para cultivos. Consecuentemente, las demandas de producción no crearon niveles suficientemente altos de tensión para conducir al desarrollo de un Estado despótico.

Este argumento particular, que será elaborado en el futuro, también se topa con dificultades que deben ser señaladas. La relación propuesta entre tensión y crecimiento sociopolítico no ha sido demostrada adecuadamente. Butzer (1967: 100), por ejemplo, argumenta que la presión de la población (tensión) podría no haber sido un problema mayor en el Egipto faraónico. A un nivel más general, estudios provisionales de la relación tensión-crecimiento se han enfocado sobre amplias categorías tecnológicas de consecusión de alimentos, pero no han sido suficientemente refinadas para ser aplicadas a situaciones prehistóricas. Por último, antes de que la relación pueda ser probada, la tensión debe ser rigurosamente definida y medida.

Suponiendo que la relación esté demostrada, los datos del área maya ofrecen varias contradicciones aparentes que requieren explicación. La sugerencia de la presencia de agricultura intensiva en campos le. vantados en el Valle del Hondo, alrededor del año 1400 a. G., es 
confusa en cuanto a que está generalmente aceptado que las tierras bajas no estaban densamente pobladas, y que el desarrollo sociopolít1co era rudimentario en esa época. Posiblemente esta situaciós pueda ser explicada en términos de tensión creada artificialmente, cuando, por alguna razón desconocida, los mayas del Hondo prefirieron in. tensificar la producción a lo largo del río en vez de extenderse fuera de éste hacia los excelentes suelos de tipo mollisols de las tierras elevadas adyacentes (Hammond, 1978; Turner y Harrison, 1978: 355 . 61; Dahlin, 1979) ; pero también los mayas bordearon algunas de las tierras limítrofes de Mesoamérica, de los Valles de' Ulúa y Chamele. cón de Honduras, y no las ocuparon aun durante épocas de supuesta alta tensión en el periodo Clásico Tardío. Los factores que evitaron esta expansión horizontal agrícola y sus implicaciones sociopolíticas requieren investigación.

La complejidad de las respuestas sociopolíticas dadas por varios pueblos a cualquier estímulo está frecuentemente enmascarada en los intentos por ofrecer explicaciones. EI enfoque basado solamente en esos ejemplos de desarrollo temprano del Estado, tiende a fortalecer los argumentos de tensión, irrigación o cualquier otra causa única, porque son excluidos ejemplos en los cuales tal desarrollo no se presenta. Una investigación más cuidadosa deberá considerar por qué el desarrollo sociopolítico estuvo ausente en esas circunstancias que, aparentemente, caben en las situaciones ambientales o económicas descritas en las diversas tesis. Por ejemplo, ¿por qué no todas las poblaciones situadas a lo largo de ríos exóticos adoptaron la irrigación y desarrollaron una organización estatal? ¿Puede ser demostrada la tensión en ciertas zonas en las cuales la población no obtuvo altos niveles de desarrollo sociopolítico? Tal atención podría revelar que las respuestas sociopolítica a las variables ambientales y económicas tam. bién incluyen un factor cultural, tal como la perseverancia de los antioquianos descritos como económicamente distintos de otros colombianos (Parsons, 1974),

El argumento presentado aquí se enfoca solamente sobre la tensión agrícola como un estimulante del desarrollo sociopolítico. Tal enfoque no relega el posible significado de otros factores económicos ( 0 , como se ha sugerido, factores culturales) que podrían interactuar con, o actuar independientemente de, las tensiones agrícolas para crear el desarrollo en cuestión. Realmente es dudoso que un cierto factor económico pueda explicar el crecimiento sociopolítico entre todas las civilizaciones tempranas. Esto es de utilidad, sin embargo, para aproximarse a un problema complejo, aislando y examinando sus diferentes componentes, mientras la totalidad de las implicaciones no sea 
examinada. En este sentido, todos los estudiosos de la prehistoria mesoamericana están en deuda con los enfoques teóricos presentados y desarrollados por Sanders y por Price.

\section{Addenda}

Posteriormente a la terminación y entrega de este trabajo en el "Simposio sobre Agro-sistemas Mexicanos: Pasado y Presente" en el XLIII Congresa Internacional de Americanistas (Vancouver, 1979), ha aparecido un número importante de argumentos y descubrimientos relacionados con la discusión. Sanders (1979) y Turner y Harrison (1979) han intercambiado puntos de vista sobre la validez de las evidencias agrícolas presentadas en el libro Pre-Hispanic Maya Agriculture (Harrison y Turner, 1978, y Sanders, Parsons y Stanley, 1979) y han ofrecido nuevos puntos de vista acerca del desarrollo del Estado en la Guenca de México. Más importante todavía es la aparición de una serie de nuevas evidencias en el área maya. Campos levantados y canales han sido reportados por Kirke a lo largo del río Belice (1980), y campos con canales han sido localizados en Lamanai (John Lambert, comunicación personal) y en Cerros (Friedel y Scarborough, s.f.) en Belice, R. E. W. Adams (comunicación personal) y sus colegas, apoyados en el Jet Propulsion Laboratory (Pasadena, California), han descubierto la probable existencia de cerca de $28000 \mathrm{kms}^{2}$ de campos y canales en Belice y en el Petén, Guatemala, utilizando sofísticadas técnicas de radar. El Pulltrouser Swamp Project (Turner, Harrison et al., s.f.) ha proporcionado la verificación en tierra de las depresiones encontradas por radar; esto es, algunos de los patrones descritos por fotografía aérea son, en realidad, campos levantados y canales mayas.

Los estudios pedológicos en las Islas Albión, Belice (Antione, Skarie y Bloom, s.f.) han puesto en tela de juicio la interpretación de que cerca de San Antonio se encuentran campos levantados (ver Puleston, 1977) y sugieren que los elementos en cuestión son campos canalizados (una red campo/canal sin grandes campos levantados). Esta evidencia ha permitido a Mary Pohl (comunicación personal) dudar de la existencia de campos levantados ahí durante el formativo, y sugiere que las fechas tempranas de radiocarbono del canal de San Antonio pueden fechar los elementos pero no los campos levantados sobre ellos.

Los diferentes trabajos sugieren fuertemente: 1) que la gran cantidad de muestras rectilíneas en tierra, identificadas de varias formas en los hábitats ribereños y en las depresiones (bajos y pantanos), en las tierras bajas mayas centrales, son campos levantados, canales y 
campos canalizados por manufactura maya; 2) los rasgos naturales en tierra de tamaño y forma similar a los campos no están ideados en esos hábitats; 3) que muchos de los campos mayas parecen haber sido construidos en medios que fueron hidrológicamente semejantes a los que tenían Xochimilco y Chalco en la época prehispánica; 4) que el manejo de las zonas ribereñas por los mayas, o el uso de canales para alguna función todavía desconocida, pudo haber sido tan tem. prana como del Formativo, pero que los campos levantados, de manera más amplia, fue un hecho que probablemente se dio del Formativo Tardío al Clásico. Para mayores detalles el lector puede consultar la bibliografía.

Finalmente, esta Addenda fue enviada como respuesta de mi punto de vista a la evidencia de que la agricultura maya no ha recibido la consideración apropiada en varios trabajos teóricos. Investigaciones subsecuentes me han permitido un mejor entendimiento de la tesis hidráulica y sus elaboraciones, en particular, la comparación entre varios conjuntos de datos utilizados para apoyar, o no, dicha tesis (Hunt y Hunt, 1976), así como el desarrollo posterior de argumentos alternos.

Así, la discusión teárica presentada deberá ser considerada sola. mente como un bosquejo. Se está elaborando una discusión más refinada del tema.

\section{BIBLIOGRAFIA}

AdAMs, RoBert MaC.

1960 "Early Givilization, Subsistence, and Environment", City Invincible: $A$ Symposium on Urbanization and Cultural Development in the Ancient Near East. G. H. Kraeling y R. McC. Adams, eds., University of Chicago Press. Chicago.

Antoine, P. P.; R. L. Skarie y P. R. Bloom

s/f "The Origin of Raised Fields near San Antonio, Belize: An Alternative Hypothesis", trabajo presentado en la Memorial Conference to Dennis E. Puleston. Minneapolis, 1979.

Bagr, Whliams y Bruge B. Hanshaw

1967 "Hydrogeology of the Northern Yucatan Peninsula, Mexico", Guide Book, Field Trip to Peninsula Yucatan: 64-78. A. E. BOSERUP, ESTER Wiedie, compiler. New Orleans Geological Society. New Orleans.

1965 The Conditions of Agricultural Growth. Aldine Pub., Chicago Brookfield, Harold C.

1962 "Local Study and Comparative Method: An Example from Central New Guinea", Annals of the Association of American Geographers, 52: 242-54. 
1972 "Intensification and Disintensification in Pacific Agriculture: A Theoretical Approach", Pacific View point, 13: 30-48.

Brookfield, Harold C. y Paula Brown

1963 Struggle for Land, Agriculture and Group Territories Among the Chimibu of the New Guinea Highlands. Oxford University Press. Londres.

Butzer, KarL W.

1976 Early Hydraulic Civilization in Egypt. University of Chicago Press. Chicago.

Garneiro, Robert L.

1970 "A Theory of the Origin of State". Science, 169: 733-738.

DAHLIN, BRUCR

1979 "Cropping Cash in the Maya Protoclassic", Maya Archaeology and Ethnohistory, 21-37, N. Hammond y G. R. Willey, eds., University of Texas Press. Austin.

Denevan, W. M. y B. L. Turner II

1974 "Forms, Functions and Associations of Raised Fields in the Old EATON, JAGK D. World Tropics", Journal of Tropical Geography, 39: 24-33.

1975 "Ancient Agricultural and Farmsteads in the Rio Bec Region of Yucatan", Contributions of the University of California Archaeological Research Facility, 27: 56-82. Berkeley.

Friedri, David A. y Vernon Scarborough

1979 "Subsistence, Trade and Development of the Coastal Maya", trabajo presentado en la Memorial Conference to Dennis $\mathrm{E}$. Puleston, Mineapolis.

Gómez-Pompa, ARTuro

1978 "An Old Answer to the Future", Mazingira, 5. Pergamon Press. Oxford.

Gómez-Pompa, Arturo y Raúl Venegas

1976 La Chinampa Tropical. INIREB Informa, 5. Instituto de Investigaciones sobre Recursos Bióticos. México.

HAMMOND, NORMAN

1978 "The Myth of the Milpa: Agricultural Expansion in the Maya Lowlands", Pre-Hispanic Maya Agriculture: 23-34. P. D. Harrison y B. L. Turner II, eds. University of New Mexico Press.

Harrison, Peter D. Albuquerque.

1977 "The rise of the Bajos and the fall of the Maya", Social Process in Maya Prehistory: Studies in Honor of Sir Eric Thompson, N. Hammond, ed. Academic Press. Londres.

Harrison, Peter D. y B. L. Turner II (eds.)

1978 Pre-Hispanic Maya Agriculture. University of New Mexico Press. Albuquerque.

Healy, Paul F., D. Draper, G. van Waarden y J. Awe

1979 "Ancient Maya Agricultural Terraces of the Cayo District. Belize: A Preliminary Report", trabajo presentado en el Symposium on Pre-Columbian Intensive Agriculture: New Research and New Perspectives, XLIII International Congress of American-

Hole, Frank ists, Vancouver.

1966 "Investigating the Origin of Mesopotamian Civilization", Science, 153: 605-611.

Hunt, Robert C. y Eva Hunt

1976 "Canal Irrigation and Local Social Organization", Current Anthropology, 17: 389-411. 
KAPPES, WAYNE

1974 "Irrigation Development and Population Pressure", Irrigation's Impact on Society: 159-167. T. E. Downing y M. Gibson, eds., University of Arizona Press. Tucson.

KIRKe, C. M. ST. G.

1980 "Prehistoric Agriculture in the Belize River Valley", World Archaeology, 2: 281-287.

\section{LANNing, EdWard P.}

1967 Peru Before the Incas. Prentice Hall. Englewood Cliffs.

I.EACH, E. R.

1959 "Hydraulic Society in Ceylan", Past and Present, 15: 2-26.

Matheny, Ray T.

1976 "Maya Loyland Hydraulic Systems", Science, 193: 639-146.

1978 "Northern Maya Lowland Water-Control Systems". Pre-Hispanic Maya Agriculture: 185-210. P. D. Harrison y B. L. Turner II,

Millon, René eds., University of New Mexico Press, Albuquerque.

1954 "Irrigation at Teotihuacan", American Antiquity, 20: 177-180.

1957 "Irrigation Systems in the Valley of Teotihuacan", American Antiquity, 23: 160-166.

1962 Variation in Response to the Practice of Irrigation Agriculture, University of Utah, Cultivation-Arid Lands, Anthropologica] Papers, 62.

Mitchell, William P.

1973 "The Hydraulic Hypothesis: A Reappraisal", Current Anthropology, 14: 532-534.

1977 "Irrigation Farming in the Andes: Evolutionary. Implications", Peasant Livelihood, P. Halperin y J. Dow, eds. St. Martin's Press. New York.

Olson, Gerald W.

1969 Description and Data on Soils of Tikal, El Peten, Guatemala, Central America (Mimeo, 69-2, Department of Agronomy, Cornell University).

1975 Study of Soils in the Sustanining Area Around San Antonio in Northern Belize (British Honduras), (Mimeo, 75-1, Department of Agronomy, Cornell University).

Palerm, ANGei y Eric Wolf

1961 "La agricultura y el desarrollo de la civilización en Mesoamérica", Revista Interamericana de Ciencias Sociales, 2a. época 1.

Parsons, James J.

1974 "The Historical Preconditions of Industrialization Medellion Reconsidered", Latin America: Search for Geographie Explanation, Proceedings of the Conference of Latin Americanist Geographers,

Price, Barbara J. 5: 119-124. R. J. Tata, ed.

1977 "Shifts in Production and Organization: A Cluster Interaction Model", Current Anthropology, 18: 209-233.

Puleston, Dennis E.

1977 "The Art and Archaeology of Hydraulic Agriculture in the Maya Lowlands", Social Process in Maya Prehistory: Studies in Honor of Sir Eric Thompson: 449-467. N. Hammond, ed., Academic Press. Londres.

Rice, Don S.

1974 The Archaeology of British Honduras: A Review and Synthesis, Occasional Papers in Anthropology, Archaeology Series, 6. Anthropology, University of Colorado. 
Rice, Don S. y Prudence M. Rice

1978 1978. Introductory Archaeological Survey of the Central Peten Savanna (A Preliminary Report to the National Geographic Society).

SABloff, Jeremy A. y William L. Rathje

1975 "The Rise of a Maya Merchant Class", Scientific American, 233: 72-82.

SANDERS, WILLIAM T.

1972 "Population, Agricultural History, and Societal Evolution in Mesoamerica", Population Growth: Anthropological Implications: 101-153, B. Spooner, ed., M. I. T. Press, Cambridge.

1977 "Environmental Heterogeneity and the Evolution of Lowland Maya Civilization", The Origins of Maya Civilization: 287-297. R. E. W. Adams, ed., University of New Mexico Press, Albuquerque.

1979 "The Jolly Green Giant in Tenth Century Yucatan, or Fact and Fancy in Classic Maya Agriculture", Review in Anthropology, 6 (4) : 493-506.

Sanders, William T. y Barbara J. Price

1968 Mesoamerica, The Evolution of a Civilization. Random House. New York.

Sanders, William T., Jeffery R. Parsons y Robert S. Santley

1979 The Basin of Mexico, Ecological Processes in The Evolution of a Civilization. Academic Press. New York.

Stemens, Alfred $\mathrm{H}$.

1977 "Some Patterns Seen from the Air". Journal of Belizean Affairs, 5: 5-21.

1978 "Karst and the Pre-Hispanic Maya in the Southern Lowlands", Pre-Hispanic Maya Agriculture: 117-143. P. D. Harrison y B. L. Turner II, eds., University of New Mexico Press, Albuquerque.

Sirmens, Alfred H. y Dennis E. Puldeston

1972 "Ridged Fields and Associated Features in Southern Campeche: New Evidence on the Lowlands Maya", American Antiquity. 37: 228-239.

Simmons, Charles S., José M. Tarano y José H. Pinto Z.

1959 Clasificación de reconocimiento de los suelos de la República de Guatemala. Ministerio de Agricultura, Instituto Agropecuario Nacional, Servicio Cooperativa Inter-Americana de Agricultura. Guatemala.

STEWARd, JUliaN H.

1949 "Cultural Causality and Law: A Trial Formulation of the Development of Early Civilization", American Anthropologist, 51: $1-27$.

TURNER, B. L., II

1974 "Prehistoric Intensive Agriculture in the Mayan Lowlands", Science, 185: 118-124.

1978a "The Development and Demise of the Swidden Thesis of Maya Agriculture", Pre-Hispanic Maya Agriculture: 13-22. P. D. Harrison y B. L. Turner II, eds., University of New Mexico Press. Albuquerque.

1978b "Ancient Agricultural Land Use in the Central Maya Lowlands", Pre-Hispanic Maya Agriculture: 163-183. P. D. Harrison y B. L. Turner II, eds., University of New Mexico Press, Albuquerque. 
1979 "Prehispanic Terracing in the Central Maya Lowlands: Problems of Agricultural Intensification", Maya Archaeology and Ethnohistory: 101-115. N. Hammond y G. R. Willey, eds., University of Texas Press, Austin.

Turner, B. L., II y Peter D. Harrison

1978 "Implications from Agriculture for Maya Prehistory", Pre-Hispanic Maya Agriculture: 337-373. P. D. Harrison y B. L. Turner II, eds., University of New Mexico Press, Albuquerque.

1979 "Comment on William T. Sander's Review of Pre-Hispanic Maya Agriculture", Reviews in Anthropology, 6 (4): 544-555.

Turner, B. L., II y Williaw G. Johnson

1978 Ecology and Subsistence in the Copan Region, Honduras, (Preliminary Report \# 1 of the Human Ecology Section of the. 1977 N. S. F.-Peabody Museum, Harvard University, Copan Valley Project.

1979 "A Maya Dam in the Copan Valley, Honduras", American Antiquity, 44 (2): 299-305.

Turner, B. L., II, Robert Q. Hanham y Anthony V. Portararo

1977 "Population Pressure and Agricultural Intensity", Annals of the Association of American Geographers, 67: 384-396.

Turner, B. L., II, et al.

1980 Maya Raised-Field Agriculture and Settlement at Pulltrouser Swamp, Northern Belize. (Report of the 1979-80, University of Oklahoma-National Science Foundation, Pulltrouser Swamp Project.

Vlemcr, David T., Sylvia Garza de González, y Edward B. Kurjagk

1978 "Contemporary Farming and Ancient Maya Settlements: Some Disconcerting Evidence", Pre-Hispanic Maya Agriculture: 211223. P. D. Harrison y B. L. Turner II, eds., University of New Mexico Press, Albuquerque.

WAdDELI, ERIC

1972 The Mound Builders. University of Washington Press. Seattle.

West, Robert G. y Pedro Armillas

1950 "Las Chinampas de México", Cuadernos Americanos, 50: 165-82.

WILKen, Gene

1977a "Integration Forest and Small-Scalle Systems in Middle America", Agro-Ecosystems, 3: 291-302.

1977b "Management of Productive Space in Traditional Farming", Actes du XLII Congres International des Americanistes (París, 1976), 2: 409-419.

WitTfogel, KaRL A.

1957 Oriental Despotism: A Comparative Study of Total Power. Yale University Press. New Haven.

1972 "The Hydraulic Approach to Pre-Spanish Mesoamerica", The Prehistory of the Tehuacan Valley, Chronology and Irrigation, 4: 59-80, F. Johnson, ed., University of Texas Press, Austin. 